\title{
Variations in the WNK1 gene modulates the effect of dietary intake of sodium and potassium on blood pressure determination
}

\author{
Yuko Osada $^{1}$, Rie Miyauchi ${ }^{2}$, Toshinao Goda ${ }^{2}$, Nobuhiko Kasezawa ${ }^{3}$, Hiromi Horiike ${ }^{1}$, Mariko Iida ${ }^{1}$, \\ Satoshi Sasaki ${ }^{4}$ and Kimiko Yamakawa-Kobayashi ${ }^{1}$
}

WNK lysine-deficient protein kinase 1 (WNK1) is a member of the WNK family of serine/threonine kinases with no lysine (K), and these kinases have been implicated as important modulators of salt homeostasis in the kidney. It is well known that high dietary sodium and low dietary potassium have been implicated in the etiology of increased blood pressure. However, the blood pressure response to dietary sodium and potassium intake varies considerably among individuals. In this study, we have detected that the haplotypes of the WNK1 gene are associated with blood pressure variations in the general Japanese population. In addition, we investigated the interactions between the haplotypes of the WNK1 gene and dietary sodium and potassium intake for determining inter-individual variations in blood pressure. Our data support the hypothesis that part of the variation in blood pressure response to dietary sodium and potassium intake among individuals can be explained by variations in the WNK1 gene. Journal of Human Genetics (2009) 54, 474-478; doi:10.1038/jhg.2009.64; published online 17 July 2009

Keywords: blood pressure; haplotype; potassium; sodium; WNK1

\section{INTRODUCTION}

Essential hypertension is one of the most common diseases of the modern world. High blood pressure or hypertension contributes to morbidity and mortality from stroke, coronary heart disease and endstage renal disease. ${ }^{1}$ Both family and epidemiological studies have suggested that $20-50 \%$ of blood pressure variation that exists within a population is of genetic origin;,3 however, the genes responsible for this variation in blood pressure have not been fully elucidated.

Blood pressure is maintained by a complex network, including renal, neuronal, endocrine and vascular mechanisms. Therefore, blood pressure is determined by complex interactions among many different genes, and is strongly influenced by environmental factors. Clinical and epidemiological studies have shown that dietary sodium intake is positively associated, and potassium intake is inversely associated, with blood pressure. ${ }^{4,5}$ In addition, the dietary sodium to potassium $(\mathrm{Na} / \mathrm{K})$ intake ratio correlates positively with blood pressure more strongly than either sodium or potassium intake alone. ${ }^{4}$ Therefore, the effects of sodium and potassium intake on blood pressure are most likely to be biologically interdependent. Furthermore, moderate sodium reduction or potassium supplementation is significantly associated with reduced blood pressure. However, the blood pressure response to dietary sodium and potassium intake seems to vary considerably among individuals. ${ }^{6}$
The WNK lysine-deficient protein kinase 1 (WNK1) and WNK4 genes are expressed in the distal nephron, specifically in the distal convoluted tubule and the connecting tubule and collecting duct. ${ }^{7}$ WNK1 and WNK4 have been implicated as important modulators of salt homeostasis, regulating the balance between renal sodium reabsorption and potassium excretion. ${ }^{8-10}$ Mutations in WNK1 or WNK4 genes cause a familial disorder, pseudohypoaldosteronism type II, which is a rare, autosomal dominant disease characterized by hypertension and elevated serum potassium levels with normal renal function. ${ }^{7}$ Therefore, the possibility has been proposed that common genetic variants in both WNK1 and WNK4 affect blood pressure variations and/or susceptibility to essential hypertension. ${ }^{11,12}$ Indeed, several studies have indicated associations between the common variants of WNK1 and WNK4 genes and blood pressure levels. ${ }^{11,13-16}$ However, the functional common variants that directly affect the activity of WNK1 or WNK4 have not been identified.

WNK1 is expressed in multiple splicing variants. At least two transcripts, a kidney-specific short form of WNK1 (KS-WNK1) and a more ubiquitous long form (L-WNK1), have been produced by alternative splicing of the WNK1 gene in the kidney. ${ }^{17,18}$ Recent studies have suggested that the ratio of L-WNK1 to KS-WNK1 is important for the regulation of renal sodium reabsorption and potassium secretion. ${ }^{12}$ Furthermore, it has been proposed that the

\footnotetext{
'Laboratory of Human Genetics, School of Food and Nutritional Sciences, Graduate School of Nutritional and Environmental Sciences, Global COE Program, University of Shizuoka, Shizuoka, Japan; ${ }^{2}$ Laboratory of Nutritional Physiology, School of Food and Nutritional Sciences, Graduate School of Nutritional and Environmental Sciences, Global COE Program, University of Shizuoka, Shizuoka, Japan; ${ }^{3}$ Department of Data Managements for Health Evaluation and Promotion, Shizuoka Medical Center, Shizuoka, Japan and ${ }^{4}$ Department of Social and Preventive Epidemiology, School of Public Health, The University of Tokyo, Tokyo, Japan Correspondence: Dr K Yamakawa-Kobayashi, Laboratory of Human Genetics, School of Food and Nutritional Sciences, University of Shizuoka, Shizuoka 422-8526, Japan. E-mail: kobayasi@u-shizuoka-ken.ac.jp
}

Received 20 March 2009; revised 31 May 2009; accepted 22 June 2009; published online 17 July 2009 
molecular switch for the splicing of L-WNK1 and KS-WNK1 is affected by the amount of dietary potassium intake. ${ }^{19-21}$ In this study, we investigated whether the common variations in the WNK1 gene are potential contributors to individual variations in blood pressure. Furthermore, we analyzed the interactions between the common WNK1 variants and dietary potassium and sodium intake for determining inter-individual variations in blood pressure.

\section{MATERIALS AND METHODS}

\section{Participants}

The participants were 691 randomly selected, apparently healthy Japanese men (mean age \pm s.d., $53.7 \pm 5.1$ years; age range, $45-65$ years), who visited a medical center for routine medical checkups. All participants provided written informed consent, and the study was approved by the Ethics Committee of the University of Shizuoka. Men taking antihypertensive drugs were excluded from the study. After overnight fasting, blood samples were collected from each participant. Resting blood pressure level was measured using a mercury sphygmomanometer in participants who were in the sitting position after at least $10 \mathrm{~min}$ of rest. Of the 691 men, 97 were diagnosed with hypertension according to the standard WHO (World Health Organization) criteria (systolic blood pressure (SBS) $\geqslant 140 \mathrm{~mm} \mathrm{Hg}$ and/or diastolic blood pressure (DBP) $\geqslant 90 \mathrm{~mm} \mathrm{Hg}$ ) by a physician. The clinical characteristics of the participants are shown in Table 1.

\section{Sodium and potassium intake assessment}

The levels of dietary sodium, potassium and alcohol intake were estimated using a brief-type self-administered diet history questionnaire (BDHQ). ${ }^{22,23}$ The BDHQ was developed on the basis of the self-administered diet history

\section{Table 1 Characteristics of the study participants}

\begin{tabular}{lc}
\hline & $\mathrm{n}=691$ (615) \\
\hline Age (years) & $53.7 \pm 5.1$ \\
SBP (mm Hg) & $120.6 \pm 15.6$ \\
DBP (mm Hg) & $76.7 \pm 11.6$ \\
Body mass index (kg m ${ }^{-2}$ ) & $23.5 \pm 2.8$ \\
Total cholesterol (mg per $100 \mathrm{ml}$ ) & $211.4 \pm 31.8$ \\
HDL cholesterol (mg per $100 \mathrm{ml})$ & $56.7 \pm 16.3$ \\
Triglyceride (mg per $100 \mathrm{ml})$ & $140.5 \pm 104.3$ \\
Diabetes mellitus (\%) & 2.6 \\
Current smoking (\%) & $2085 \pm 570$ \\
Total energy intake (kcal per day) & $4522 \pm 1352$ \\
Sodium intake (mg per day) & $2305 \pm 795$ \\
Potassium intake (mg per day) & $2.03 \pm 0.41$ \\
Sodium to potassium intake ratio & $28.0 \pm 32.0$ \\
Alcohol intake (g per day) & \\
\hline Abbreviations: DBP, diastolic blood pressure; HDL, high-density lipoprotein; & SBP, systolic blood \\
pressure. & \\
Data are expressed as mean \pm s.d. or percentages. & \\
Number of participants whose dietary intake data were available is in parenthesis.
\end{tabular}

questionnaire (DHQ), which has been validated using three different standard methods for dietary assessment. ${ }^{24}$ The BDHQ and DHQ were designed to obtain dietary habits for the previous month for total energy and 38 nutrients including sodium and potassium. ${ }^{22-24}$ Dietary data were available for 615 participants $(89.0 \%)$ in this study.

\section{DNA analysis}

Genomic DNA was isolated from peripheral blood leukocytes using the phenol extraction method. We have determined the genotypes of five single-nucleotide polymorphisms (SNPs) of the WNK1 gene (rs2286007, rs880054, rs956868, rs12828016 and rs2255390) by PCR-restriction fragment length polymorphism analysis. We have selected these SNPs on the basis of the public NCBI dbSNP database (http://www.ncbi.nlm.nih.gov/SNP/). At first, we have analyzed the heterozygosity and genotyping success rate for 12 SNPs (rs11554421, rs2369402, rs2240282, rs2286006, rs2286007, rs880054, rs9804992, rs16928108, rs956868, rs12301299, rs12828016 and rs2255390) in a pilot study comprising 96 participants, and selected 3 non-synonymous SNPs (rs2286007, rs956868 and rs12828016) with a relatively high heterozygosity (minor allele frequency $(\mathrm{MAF})>0.10$ ) and two intronic SNPs (rs880054 and rs2255390) with high heterozygosity (MAF $>0.45$ ). The genotyping success rate for each of these five SNPs is $>98 \%$. The genomic structure of the WNK1 gene and locations of these five SNPs are shown in Figure 1.

\section{Statistical analysis}

The relationships between blood pressure levels and each genotype or haplotype of the WNK1 gene were analyzed by multiple linear regression analysis incorporating age, body mass index (BMI), smoking and alcohol consumption as covariates. Statistical analyses were carried out using the JMP software package (SAS Institute, Cary, NC, USA). $P<0.05$ was considered statistically significant. No adjustment for multiple testing was made. The coefficients of linkage disequilibrium (LD) $\left(\left|D^{\prime}\right|\right.$ and $\left.r^{2}\right)$ were calculated using the SNPAlyze program (Dynacom, Yokohama, Japan). The haplotypes and their frequencies were estimated by the maximum-likelihood method with an EM-based algorithm using the SNPAlyze program.

\section{RESULTS}

We analyzed the relationships between the genotypes of five SNPs (rs2286007, rs880054, rs956868, rs12828016 and rs2255390) in the WNK1 gene and blood pressure levels. Statistically significant associations were observed between the genotypes of three SNPs (rs880054, rs956868 and rs12828016) and SBP and/or DBP levels in the multiple linear regression analysis adjusted for age, BMI, smoking and alcohol consumption as covariates. The A allele (rs880054, A $>\mathrm{G}$ polymorphism in intron 10), the Thr allele (rs956868, Pro 1056 Thr polymorphism in exon 13) and the Met allele (rs12828016, Met1808Ile polymorphism in exon 21) were associated with increased blood pressure with gene dosage effects. On the other hand, the genotypes of the remaining two SNPs (rs2286007 and rs2255390) were not associated with blood pressure levels (Table 2).

These five SNPs were in LD with each other $\left(\left|D^{\prime}\right|>0.85 \mid\right)$, in particular the genotypes of the $A>G$ polymorphism in intron 10 (rs880054) and the Met1808Ile polymorphism (rs12828016) were

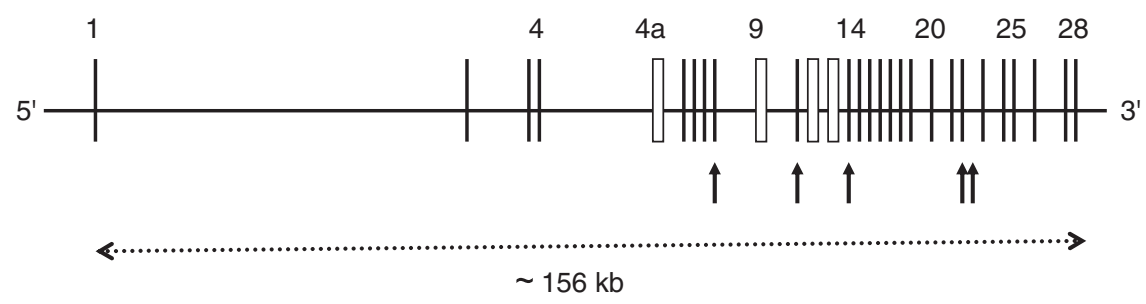

Figure 1 Genomic structure of the WNK1 gene and location of the genotyped single-nucleotide polymorphisms (SNPs). Exons are indicated by the vertical bars and alternatively spliced exons by the white boxes. The locations of the five genotyped SNPs are indicated by arrows. 
identical in almost all participants (98.0\%). The pairwise LD values of $\left|D^{\prime}\right|$ and $r^{2}$ are shown in Table 3. There are four common haplotypes with frequencies of $>5 \%$ that account for $97 \%$ of all chromosomes in our participants (Table 4 ).

The SBP level in carriers of haplotype 3 (Thr-A-Thr-Met-G) was higher than that in noncarriers of this haplotype $(P=0.025)$ (Table 5$)$.

Table 2 Relations between genotypes of five SNPs in WNK1 and the SBP and DBP levels

\begin{tabular}{|c|c|c|c|c|c|}
\hline$d b S N P$ & Region & Genotype & $n$ & $S B P(m m H g)$ & $D B P(m m ~ H g)$ \\
\hline rs2286007 & Exon 8 & Thr/Thr & 579 & $120.6 \pm 16.0$ & $76.6 \pm 11.8$ \\
\hline \multirow[t]{2}{*}{ (Thr665Ile) } & & Thr/lle & 100 & $120.9 \pm 14.0$ & $78.0 \pm 11.1$ \\
\hline & & Ile/lle & 10 & $\begin{array}{c}112.2 \pm 14.4 \\
P=0.13\end{array}$ & $\begin{array}{c}69.8 \pm 12.4 \\
P=0.16\end{array}$ \\
\hline rs880054 & Intron 10 & AA & 279 & $122.6 \pm 17.3$ & $78.0 \pm 12.1$ \\
\hline \multirow[t]{3}{*}{$(A>G)$} & & $A G$ & 319 & $119.8 \pm 14.4$ & $76.3 \pm 11.5$ \\
\hline & & GG & 88 & $116.6 \pm 13.8$ & $74.1 \pm 10.4$ \\
\hline & & & & $P=0.0088$ & $P=0.045$ \\
\hline rs956868 & Exon 13 & Pro/Pro & 505 & $119.6 \pm 15.7$ & $76.1 \pm 11.5$ \\
\hline \multirow[t]{3}{*}{ (Pro1056Thr) } & & Pro/Thr & 171 & $122.8 \pm 15.5$ & $78.3 \pm 12.3$ \\
\hline & & Thr/Thr & 15 & $125.3 \pm 14.0$ & $78.3 \pm 9.8$ \\
\hline & & & & $P=0.032$ & $P=0.20$ \\
\hline rs12828016 & Exon 21 & Met/Met & 283 & $122.7 \pm 17.1$ & $78.1 \pm 12.0$ \\
\hline \multirow[t]{3}{*}{ (Met1808Ile) } & & Met/Ile & 312 & $119.8 \pm 14.3$ & $76.3 \pm 11.5$ \\
\hline & & Ile/lle & 92 & $117.1 \pm 13.9$ & $74.3 \pm 10.7$ \\
\hline & & & & $P=0.0096$ & $P=0.043$ \\
\hline rs2255390 & Intron 22 & $\mathrm{GG}$ & 184 & $119.5 \pm 14.0$ & $75.8 \pm 11.6$ \\
\hline \multirow[t]{3}{*}{$(G>A)$} & & $\mathrm{GA}$ & 331 & $120.6 \pm 15.4$ & $76.9 \pm 11.3$ \\
\hline & & $A A$ & 173 & $121.3 \pm 17.8$ & $77.3 \pm 12.6$ \\
\hline & & & & $P=0.47$ & $P=0.44$ \\
\hline
\end{tabular}

Abbreviations: BMI, body mass index; DBP, diastolic blood pressure; SBP, systolic blood pressure; SNP, single-nucleotide polymorphism.

Values are shown as mean \pm s.d.

$P$-values were calculated by multiple linear regression analyses incorporating age, BMI, current

smoking and alcohol intake as covariates. Statistically significant $P$ values are indicated in bold.
On the other hand, the SBP and DBP levels of carriers of haplotype 2 (Thr-G-Pro-Ile-G) were lower than those in noncarriers of this haplotype ( $P=0.017, P=0.0083$, respectively) (Table 5 ). These data indicate that haplotype 3 is associated with increased blood pressure levels, whereas haplotype 2 is associated with decreased blood pressure levels.

Next, to examine the interaction between the haplotypes of the WNK1 gene and dietary sodium and potassium intake for determining blood pressure levels, we classified the participants into two groups according to population median for the $\mathrm{Na} / \mathrm{K}$ intake ratio. As expected, of the total participants, the SBP and DBP levels in the group with a high $\mathrm{Na} / \mathrm{K}$ intake ratio were higher than those of the group with a low $\mathrm{Na} / \mathrm{K}$ intake ratio $(P=0.0085, P=0.013$, respectively) (Table 6). These significant differences in blood pressure levels caused by the dietary $\mathrm{Na} / \mathrm{K}$ intake ratio were observed in carriers of both haplotype 1 (Thr-A-Pro-Met-A) and haplotype 2. Among the carriers of haplotype 1 and haplotype 2, the SBP or DBP levels in the group with a high $\mathrm{Na} / \mathrm{K}$ intake ratio were significantly higher than those in the group with a low $\mathrm{Na} / \mathrm{K}$ intake ratio $(P=0.019, P=0.025$, respectively) (Table 6). On the other hand, the blood pressure levels in carriers with haplotype 3 were similar among both the high and low $\mathrm{Na} / \mathrm{K}$ intake ratio groups. These findings suggest that the genetic differences in the WNK1 gene influence the variations in blood pressure response to dietary sodium and potassium intake.

\section{DISCUSSION}

Although epidemiological and genetic studies have shown a high heritability of blood pressure variation, identification of the genes responsible for essential hypertension has been very difficult owing to the complexity and heterogeneity of the trait. The regulation of net renal sodium reabsorption and potassium excretion in the kidney is a major determinant of blood pressure. ${ }^{3,25}$ Furthermore, the most widely studied environmental aspect of hypertension is the relationship between sodium and potassium intake. ${ }^{4}$ However, the blood pressure response to dietary sodium and potassium intake is believed to vary considerably among individuals. Genetic factors may also have

Table 3 The pairwise linkage disequilibrium (LD) values of $\left|D^{\prime}\right|$ (upper) and $r^{2}$ (lower)

$\left|D^{\prime}\right|$

\begin{tabular}{|c|c|c|c|c|c|}
\hline$r^{2}$ & rs2286007 (Thr665/le) & $r s 880054(A>G)$ & rs956868 (Pro1056Thr) & rs12828016 (Met1808/le) & $r s 2255390(G>A)$ \\
\hline rs2286007 (Thr665Ile) & & 0.87 & 1 & 0.89 & 0.94 \\
\hline rs880054 (A>G) & 0.13 & & 1 & 0.99 & 0.98 \\
\hline rs956868 (Pro1056Thr) & 0.016 & 0.095 & & 1 & 0.97 \\
\hline rs12828016 (Met1808Ile) & 0.14 & 0.96 & 0.095 & & 0.98 \\
\hline rs2255390 $(G>A)$ & 0.083 & 0.54 & 0.16 & 0.53 & \\
\hline
\end{tabular}

$\left|D^{\prime}\right|$, standardized disequilibrium coefficient.

$r^{2}$, squared correlation coefficient.

Table 4 Estimated WNK1 haplotypes and frequencies in the general Japanese population

\begin{tabular}{|c|c|c|c|c|c|c|}
\hline & rs2286007 & rs880054 & rs956868 & rs12828016 & rs2255390 & Frequency \\
\hline & Thr665/le & $A>G$ & Pro1056Thr & Met1808/le & $G>A$ & $(\%)$ \\
\hline Haplotype 1 & Thr & A & Pro & Met & $A$ & 48.1 \\
\hline Haplotype 2 & Thr & G & Pro & Ile & $\mathbf{G}$ & 28.3 \\
\hline Haplotype 3 & Thr & A & Thr & Met & $\mathbf{G}$ & 13.8 \\
\hline Haplotype 4 & Ile & G & Pro & Ile & G & 7.8 \\
\hline
\end{tabular}

Major alleles are indicated in bold. 
Table 5 Comparison of blood pressure levels between carriers and non-carriers of each haplotype

\begin{tabular}{lcccc}
\hline & & $\mathrm{N}$ & $S B P(\mathrm{~mm} \mathrm{Hg})$ & $D B P(\mathrm{~mm} \mathrm{Hg})$ \\
\hline Haplotype 1 & $(+)$ & 488 & $120.9 \pm 16.2$ & $77.1 \pm 11.7$ \\
& $(-)$ & 180 & $119.3 \pm 14.2$ & $75.6 \pm 11.7$ \\
Haplotype 2 & & & $P=0.23$ & $P=0.19$ \\
& $(+)$ & 312 & $118.8 \pm 14.5$ & $75.3 \pm 11.1$ \\
Haplotype 3 & $(-)$ & 356 & $122.0 \pm 16.5$ & $78.0 \pm 12.0$ \\
& & & $\boldsymbol{P}=\mathbf{0 . 0 1 7}$ & $\boldsymbol{P}=\mathbf{0 . 0 0 8 3}$ \\
& $(+)$ & 173 & $123.3 \pm 15.5$ & $78.3 \pm 12.1$ \\
Haplotype 4 & & 495 & $119.5 \pm 15.6$ & $76.1 \pm 11.5$ \\
& $(+)$ & 99 & $120.3 \pm 13.9$ & $77.5 \pm 11.3$ \\
& $(-)$ & 569 & $120.5 \pm 16.0$ & $76.5 \pm 11.8$ \\
& & & $P=0.92$ & $P=0.32$
\end{tabular}

Abbreviations: BMI, body mass index; DBP, diastolic blood pressure; SBP, systolic blood pressure.

$P$-values were calculated by multiple linear regression analyses incorporating age, BMI, smoking $P$-values were calculated by multiple linear regression analyses incorporating age, BMI,
and alcohol intake as covariates. Statistically significant $P$ values are indicated in bold.

an important role not only in determining blood pressure levels but also in determining the blood pressure responses to dietary sodium and potassium intake. ${ }^{6}$

The WNK kinases and several ion transport systems in the kidney work together for maintaining sodium and potassium homeostasis, and consequently blood pressure regulation. ${ }^{8,9,12,26}$ WNK1 is expressed in two splicing variants in the kidney, namely KS-WNK1 and L-WNK1. ${ }^{17,18}$ They have remarkably different effects; KS-WNK1 antagonizes the ability of L-WNK1 to inhibit renal outer medullary $\mathrm{K}^{+}$ (ROMK) and to activate the epithelial $\mathrm{Na}$ channel $(\mathrm{ENaC})$ and $\mathrm{Na}^{+} \mathrm{Cl}^{-}$cotransporter (NCC). Therefore, a decrease in the ratio of L-WNK1 to KS-WNK1 enhances potassium secretion through ROMK and decreases sodium reabsorption through $\mathrm{ENaC}$ and NCC. ${ }^{8,12}$ Therefore, the ratio of L-WNK1 to KS-WNK1 is very important for the regulation of renal potassium secretion and sodium reabsorption. Recent studies have indicated that the expression of the mRNA and protein of KS-WNK1 is significantly increased by a high potassium diet compared with a low potassium diet in rats. ${ }^{19,20}$ These findings indicate the possibility that variations in dietary potassium intake cause reciprocal changes in the ratio of L-WNK1 to KS-WNK1 in human kidneys, and consequently in the regulation of renal potassium secretion and sodium reabsorption.

In this study, we have detected that the haplotypes of the WNK1 gene that are associated with individual variations of blood pressure in the general Japanese population, and that the individual blood pressure response to dietary sodium and potassium intake differs among the WNK1 haplotypes. We speculate that the L-WNK1 and KS-WNK1 expression ratio in the kidney is affected not only by dietary potassium intake but also by variations in the WNK1 gene. The possibility exists that KS-WNK1 expression is not increased in carriers of haplotype 3 when their potassium intake is increased. On the other hand, for the carriers of haplotype 1 or haplotype 2, the expression ratio of L-WNK1 to KS-WNK1 in the kidney may be modulated by the amount of dietary sodium and potassium intake.

The possibility exists that some of the individual differences in blood pressure response to dietary sodium and potassium intake are represented by variations in the WNK1 gene. However, at present, we have no direct evidence that such variations in the WNK1 gene cause alterations in the expression ratio of L-WNK1 and KS-WNK1 and/or
Table 6 Interactions between WNK1 haplotypes and sodium to potassium $(\mathrm{Na} / \mathrm{K})$ intake ratio

\begin{tabular}{|c|c|c|c|c|c|c|}
\hline & & \multicolumn{2}{|c|}{ Low $\mathrm{Na} / \mathrm{K}$ intake group } & \multicolumn{2}{|c|}{ High $\mathrm{Na} / \mathrm{K}$ intake group } & \multirow[b]{2}{*}{ P-value } \\
\hline & & $n$ & $S B P(m m ~ H g)$ & $n$ & $S B P(m m ~ H g)$ & \\
\hline \multicolumn{2}{|l|}{ Total participants } & 309 & $119.0 \pm 15.7$ & 306 & $122.0 \pm 15.6$ & 0.0085 \\
\hline \multirow[t]{2}{*}{ Haplotype 1} & $(+)$ & 220 & $119.2 \pm 16.5$ & 217 & $122.7 \pm 15.8$ & 0.019 \\
\hline & $(-)$ & 80 & $118.1 \pm 13.2$ & 73 & $119.5 \pm 15.0$ & 0.32 \\
\hline \multirow[t]{2}{*}{ Haplotype 2} & $(+)$ & 141 & $117.2 \pm 14.0$ & 133 & $120.4 \pm 15.1$ & 0.057 \\
\hline & $(-)$ & 159 & $120.5 \pm 17.0$ & 157 & $123.2 \pm 16.0$ & 0.07 \\
\hline \multirow[t]{2}{*}{ Haplotype 3} & $(+)$ & 71 & $122.6 \pm 14.2$ & 79 & $123.3 \pm 16.2$ & 0.65 \\
\hline & $(-)$ & 229 & $117.8 \pm 16.0$ & 211 & $121.4 \pm 15.4$ & 0.017 \\
\hline \multirow[t]{3}{*}{ Haplotype 4} & $(+)$ & 41 & $118.5 \pm 15.0$ & 41 & $121.0 \pm 12.7$ & 0.48 \\
\hline & $(-)$ & 259 & $119.0 \pm 15.9$ & 249 & $122.1 \pm 16.1$ & 0.018 \\
\hline & & $n$ & $D B P(m m ~ H g)$ & $n$ & $D B P(m m ~ H g)$ & P-value \\
\hline \multicolumn{2}{|l|}{ Total participants } & 309 & $75.5 \pm 12.0$ & 306 & $77.9 \pm 10.9$ & 0.013 \\
\hline \multirow[t]{2}{*}{ Haplotype 1} & $(+)$ & 220 & $75.9 \pm 12.1$ & 217 & $78.3 \pm 10.9$ & 0.053 \\
\hline & $(-)$ & 80 & $74.4 \pm 11.9$ & 73 & $76.2 \pm 11.0$ & 0.26 \\
\hline \multirow[t]{2}{*}{ Haplotype 2} & $(+)$ & 141 & $73.8 \pm 11.5$ & 133 & $76.7 \pm 10.6$ & 0.025 \\
\hline & $(-)$ & 159 & $77.0 \pm 12.3$ & 157 & $78.6 \pm 11.1$ & 0.33 \\
\hline \multirow[t]{2}{*}{ Haplotype 3} & $(+)$ & 71 & $78.2 \pm 12.3$ & 79 & $77.5 \pm 11.0$ & 0.46 \\
\hline & $(-)$ & 229 & $74.7 \pm 11.8$ & 211 & $77.9 \pm 10.9$ & 0.0052 \\
\hline \multirow[t]{2}{*}{ Haplotype 4} & $(+)$ & 41 & $77.7 \pm 12.5$ & 41 & $76.7 \pm 9.2$ & 0.55 \\
\hline & $(-)$ & 259 & $75.2 \pm 11.9$ & 249 & $77.9 \pm 11.2$ & 0.0094 \\
\hline
\end{tabular}

Abbreviations: BMI, body mass index; DBP, diastolic blood pressure; SBP, systolic blood pressure.

$P$-vales were calculated by multiple linear regression analyses incorporating age, BMI, current smoking and alcohol intake as covariates. Statistically significant $P$ values are indicated in bold.

the in the activity of L-WNK1 in vivo. Further in vivo and in vitro studies are required to determine the functional changes in WNK1 and the ion transport systems in the kidney.

We found that an appropriate $\mathrm{Na} / \mathrm{K}$ intake ratio is effective in decreasing blood pressure levels for carriers of haplotype 1 or haplotype 2 in the WNK1 gene; however, this is not effective for carriers of the haplotype 3 . It will be important to gather scientific evidence of any interactions or otherwise between genetic and modifiable environmental factors such as dietary nutrient intake to establish a preventive method for common diseases, such as hypertension. If such interactions are detected, then modification of such dietary exposure may be suitable for certain population subgroups with important health consequences.

\section{ACKNOWLEDGEMENTS}

We are grateful to the participants for their participation in this study. This study was supported by the Grant-in-Aid for Scientific Research (C) (19500602) from the Japan Society for the Promotion of Science (JSPS) and the Global COE program from the Ministry of Education, Culture, Sport, Science and Technology of Japan (MEXT).

1 Lewington, S. \& Prospective Studies Collaboration. Age-specific relevance of usual blood pressure to vascular mortality: a meta-analysis of individual data for one million adults in 61 prospective studies. Lancet 360, 1903-1913 (2002).

2 Ward, R. Familial aggregation and genetic epidemiology of blood pressure. In Hypertension Pathophysiology, Diagnosis, and Management (eds. Laragh, J.H. \& Brenner, B.M.) 81-100 (Raven Press, New York, 1990).

3 Gharavi, A. \& Lifton, R. The inherited basis of blood pressure variation and hypertension. In The Metabolic and Molecular Basis of Inherited Disease, 8th edn. (eds. 
Scriver, C.R., Beaudet, A.L., Sly, W.S. \& Valle, D.) 4, 5399-5417 (McGraw Hill, New York, 2002).

4 Khaw, K. T. \& Barrett-Connor, E. The association between blood pressure, age, and dietary sodium and potassium: a population study. Circulation 77, 53-61 (1988).

5 Morris, Jr. R. C., Schmidlin, O., Frassetto, L. A. \& Sebastian, A. Relationship and interaction between sodium and potassium. J. Am. Coll. Nutr. 25, 262S-270S (2006).

6 Gu, D., Rice, T., Wang, S., Yang, W., Gu, C., Chen, C. S. et al. Heritability of blood pressure responses to dietary sodium and potassium intake in a Chinese population. Hypertension 50, 116-122 (2007).

7 Wilson, F. H., Disse-Nicodeme, S., Choate, K. A., Ishikawa, K., Nelson-Williams, C., Desitter, I. et al. Human hypertension caused by mutations in WNK kinases. Science 293, 1107-1112 (2001).

8 Huang, C. L., Yang, S. S. \& Lin, S. H. Mechanism of regulation of renal ion transport by WNK kinases. Curr. Opin. Nephrol. Hypertens. 17, 519-525 (2008).

9 Coffman, T. M. A WNK in the kidney controls blood pressure. Nat. Genet. 38, 1105-1106 (2006).

10 Lalioti, M. D., Zhang, J., Volkman, H. M., Kahle, K. T., Hoffmann, K. E., Toka, H. R. et al. Wnk 4 controls blood pressure and potassium homeostasis via regulation of mass and activity of the distal convoluted tubule. Nat. Genet. 38, 1124-1132 (2006).

11 Newhouse, S. J., Wallace, C., Dobson, R., Mein, C., Pembroke, J., Farrall, M. et al. Haplotypes of the WNK1 gene associate with blood pressure variation in a severely hypertensive population from the British Genetics of Hypertension study. Hum. Mol. Genet. 14, 1805-1814 (2005).

12 Huang, C. L., Kuo, E. \& Toto, R. D. WNK kinases and essential hypertension. Curr. Opin. Nephrol. Hypertens. 17, 133-137 (2008).

13 Kokubo, Y., Kamide, K., Inamoto, N., Tanaka, C., Banno, M., Takiuchi, S. et al. Identification of 108 SNPs in TSC, WNK1, and WNK4 and their association with hypertension in a Japanese general population. J. Hum. Genet. 49, 507-515 (2004).

14 Tobin, M. D., Raleigh, S. M., Newhouse, S., Braund, P., Bodycote, C., Ogleby, J. et al. Association of WNK1 gene polymorphisms and haplotypes with ambulatory blood pressure in the general population. Circulation 112, 3423-3429 (2005).

15 Turner, S. T., Schwartz, G. L., Chapman, A. B. \& Boerwinkle, E. WNK1 kinase polymorphism and blood pressure response to a thiazide diuretic. Hypertension 46, 758-765 (2005).
16 Tobin, M. D., Timpson, N. J., Wain, L. V., Ring, S., Jones, L. R., Emmett, P. M. et al. Common variation in the WNK1 gene and blood pressure in childhood: the Avon Longitudinal Study of Parents and Children. Hypertension 52, 974-979 (2008).

17 O'Reilly, M., Marshall, E., Speirs, H. J. \& Brown, R. W. WNK1, a gene within a novel blood pressure control pathway, tissue-specifically generates radically different isoforms with and without a kinase domain. J. Am. Soc. Nephrol. 14, 2447-2456 (2003).

18 Delaloy, C., Lu, J., Houot, A. M., Disse-Nicodeme, S., Gasc, J. M., Corvol, P. et al. Multiple promoters in the WNK1 gene: one controls expression of a kidney-specific kinase-defective isoform. Mol. Cell Biol. 23, 9208-9221 (2003).

19 Lazrak, A., Liu, Z. \& Huang, C. L. Antagonistic regulation of ROMK by long and kidneyspecific WNK1 isoforms. Proc. Natl Acad. Sci. USA 103, 1615-1620 (2006).

20 Wade, J. B., Fang, L., Liu, J., Li, D., Yang, C. L. \& Subramanya, A. R. et al. WNK1 kinase isoform switch regulates renal potassium excretion. Proc. Natl Acad. Sci. USA 103, 8558-8563 (2006).

21 O'Reilly, M., Marshall, E., Macgillivray, T., Mittal, M., Xue, W., Kenyon, C. J. et al. Dietary electrolyte-driven responses in the renal WNK kinase pathway in vivo. J. Am. Soc. Nephrol. 17, 2402-2413 (2006).

22 Sasaki, S., Yanagibori, R. \& Amano, K. Self-administered diet history questionnaire developed for health education: a relative validation of the test-version by comparison with 3-day diet record in women. J. Epidemiol. 8, 203-215 (1998).

23 Sasaki, S., Yanagibori, R. \& Amano, K. Validity of a self-administered diet history questionnaire for assessment of sodium and potassium: comparison with single 24-hour urinary excretion. Jpn. Circ. J. 62, 431-435 (1998).

24 Sasaki, S., Takahashi, T., litoi, Y., Iwase, Y., Kobayashi, M., Ishihara, J. et al. Food and nutrient intakes assessed with dietary records for the validation study of a selfadministered food frequency questionnaire in JPHC Study Cohort I. J. Epidemiol. 13, S23-S50 (2003)

25 Lifton, R. P., Gharavi, A. G. \& Geller, D. S. Molecular mechanisms of human hypertension. Cell 104, 545-556 (2001).

26 McCormick, J. A., Yang, C. L. \& Ellison, D. H. WNK kinases and renal sodium transport in health and disease: an integrated view. Hypertension 51, 588-596 (2008). 